\title{
Revisiting the role of the hepatic vein in laparoscopic liver resection
}

\author{
Daisuke Ban, Satoshi Nara, Takeshi Takamoto, Takahiro Mizui, Jun Yoshino, Minoru Esaki, Kazuaki \\ Shimada
}

Department of Hepatobiliary and Pancreatic Surgery, National Cancer Center Hospital, Tokyo 104-0045, Japan.

Correspondence to: Dr. Daisuke Ban, Department of Hepatobiliary and Pancreatic Surgery, National Cancer Center Hospital, 5-1-1 Tsukiji, Chuo-ku, Tokyo 104-0045, Japan. E-mail: dban@ncc.go.jp

How to cite this article: Ban D, Nara S, Takamoto T, Mizui T, Yoshino J, Esaki M, Shimada K. Revisiting the role of the hepatic vein in laparoscopic liver resection. Hepatoma Res 2021;7:13. http://dx.doi.org/10.20517/2394-5079.2020.104

Received: 13 Sep 2020 First Decision: 9 Nov 2020 Revised: 10 Nov 2020 Accepted: 17 Nov 2020 Published: 3 Feb 2021

Academic Editor: Ho-Seong Han Copy Editor: Cai-Hong Wang Production Editor: Jing Yu

\begin{abstract}
Laparoscopic liver resection (LLR) has expanded to include major liver resection and systematic resection as the techniques have advanced. Regardless of the oncological significance of anatomical resection, dissection of the intersegmental plane is useful in liver resection because it makes liver dissection easier and does not leave an ischemic area. In laparoscopic surgery, the hepatic vein can be exposed with less bleeding than in open surgery because bleeding can be controlled by pneumoperitoneum pressure. Therefore, the hepatic vein is a useful indicator to guide the dissected surface and to determine the depth. However, the basic technique of exposing the hepatic vein during LLR is required. The hepatic vein root can be approached using either a cranial or dorsal approach, with the dorsal approach providing the favorable view characteristic of laparoscopy. Selecting the dissection layer with the Laennec's capsule of the hepatic vein roots in mind is also a useful technique to ensure more reliable dissection of the hepatic vein. We summarize previous reports on techniques for facilitating LLR using the hepatic vein as a guide and outline the role of each hepatic vein type. Although there are many reports of procedures using the hepatic vein as a guide, the terminology of the approach awaits standardization in the future.
\end{abstract}

Keywords: Laparoscopic liver resection, laparoscopic hepatectomy, hepatic vein, anatomical landmark

\section{INTRODUCTION}

Initially limited to minor liver resections, laparoscopic liver resection (LLR) has been expanded to include major liver resection and systematic resection as techniques have advanced ${ }^{[1]}$. Although there has been

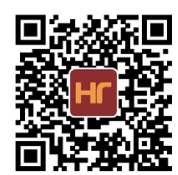


controversy about the oncological significance of anatomical hepatectomy, most surgeons would agree that minimizing vessel dissection on the intersegmental plane makes it easy to perform liver resection. In contrast with the concept of parenchyma-sparing hepatectomy, some reports suggest that hepatectomy that does not result in an ischemic area has a better prognosis ${ }^{[2,3]}$.

To make an anatomically reasonable dissection, landmarks that serve as indicators of dissection must be used. One is the root of the responsible Glisson branch and the other is the regional boundary of the liver surface. In actuality, the boundaries of the dominant region are not a straight line at the liver surface and are not a flat plane relative to the parenchyma. The traditional method of staining the portal vein is by its direct puncture to mark the dissection line on the liver surface, and more recently the usefulness of intraoperative visualization of the dominant region by indocyanine green (ICG) for liver dissection has been reported ${ }^{[4,5]}$. These are techniques that are not limited to laparoscopic surgery but are also common in open surgery. However, in LLR, intraoperative ultrasonography-guided puncture of the portal vein branch is technically demanding, and indigo staining is impractical. However, visualization with ICG is extremely useful and likely expected to become one of the main techniques in LLR in the future ${ }^{[6]}$.

Although boundaries are marked on the liver surface as indicators for dissection, landmark structures in the liver parenchyma during liver dissection are also needed. The hepatic veins, another important landmark, have traditionally been exposed during hepatic dissection as structures that divide the segment. Controlling hemorrhage from the hepatic veins and exposing them is an important step in liver resection. We describe the role of the hepatic vein in LLR on the basis of previous reports and our own experience.

\section{ROLE OF HEPATIC VEINS}

\section{Technique for exposing hepatic veins during LLR}

There are several benefits of laparoscopic surgery in liver resection. The most notable is the control of venous bleeding due to pneumoperitoneum. If well controlled by the equilibrium of hepatic venous and pneumoperitoneum pressures, no bleeding occurs if the pore is small ${ }^{[7]}$. Lower central venous pressure is necessary to lower the hepatic venous pressure. Low intraoperative fluid levels, low airway pressure, an elevated head position, and other controls on respiration and circulation are essential for minimizing venous bleeding in LLR. This seems to be the main reason for the results of previous large retrospective studies and randomized controlled trials that showed less blood loss in LLR compared with open liver resection ${ }^{[8,9]}$. To perform a safe and effective transection following hepatic veins, the collaboration with the anesthesiologist is of paramount importance.

One of the technical difficulties of LLR is achieving accurate 3-D spatial orientation. It is easy to become disoriented during the procedure, particularly during partial resection. For example, in the case of a partial resection of segment 8 , the dorsal margin of the tumor may not be sufficient as the resection proceeds. Observation by ultrasonography during surgery is also essential. If there is a vein on the back side that serves as a landmark for the resection line, the target hepatic vein can be included on the resection side once it is exposed, thereby ensuring that the partial resection is performed without missing the resection line [Figure 1].

It is unquestionably easier to expose the vein during laparoscopic surgery, but this requires basic techniques to avoid bleeding from the vein. As the vein is dissected from the periphery to the center, the angle of the small venous branch that confluences to the larger vein causes it to split and injure the branch. To avoid this, it is important to dissect from the center to the periphery. Honda et al. ${ }^{[10]}$ introduced a technique that divides venous injuries into two types: spit injuries, which are peripheral to central injuries, and pulledup injuries, which are central to peripheral injuries and are easier to control. The key to this method is to move the Cavitron ultrasonic surgical aspirator (CUSA) from the root to the peripheral side so that 

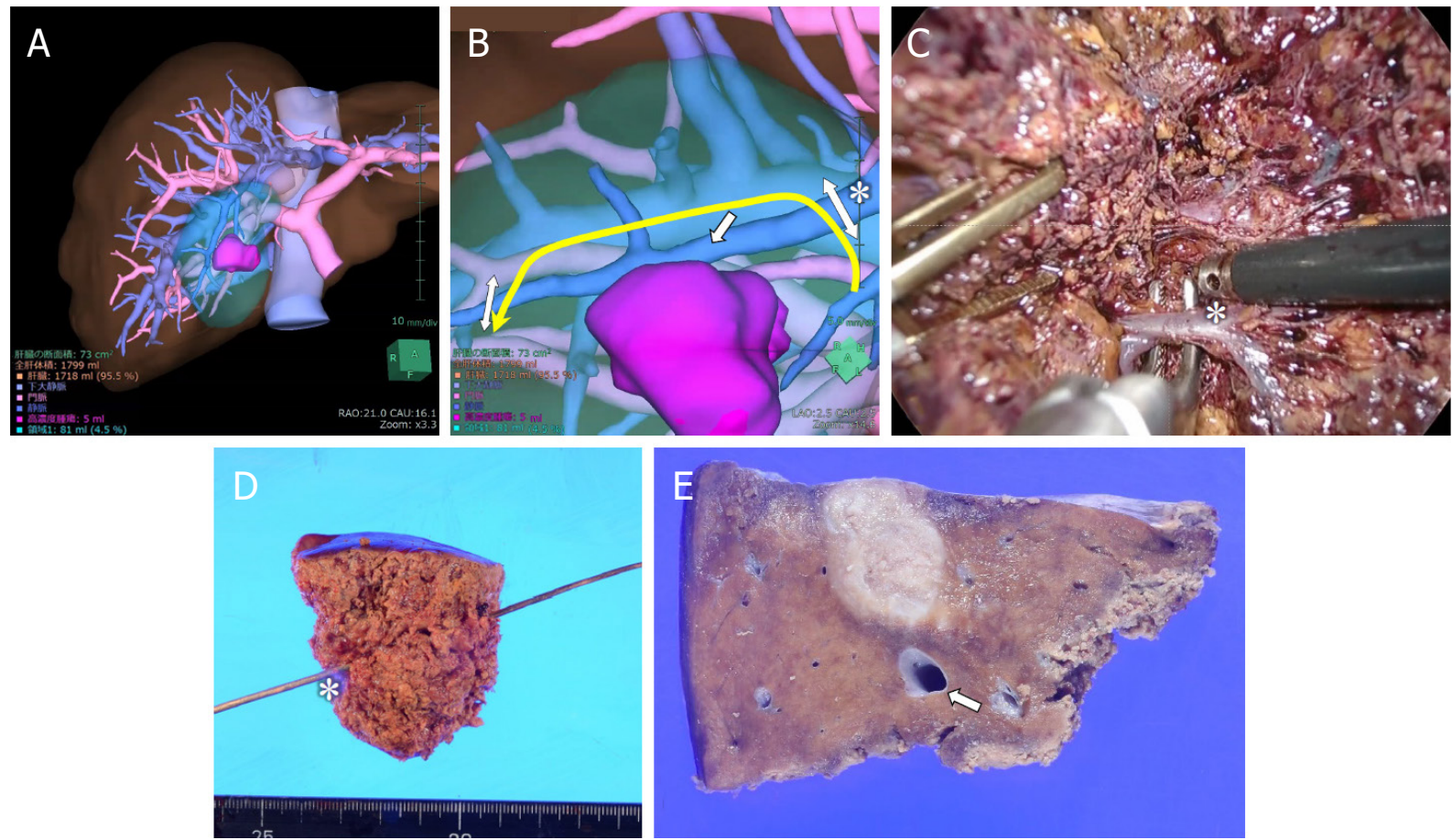

Figure 1. Laparoscopic partial liver resection of segment 5. A: preoperative simulation; $\mathrm{B}$ : the resection plan is to include the $\mathrm{MHV}$ on the dorsal side of the tumor on the resection side. White arrows indicate MHVs; $\mathrm{C}$ : the targeted MHV is encircled on the left side of the resection plane; D: the planned $\mathrm{MHV}$ is included on the resection side; $\mathrm{E}$ : the divided surface of the specimen after resection. The white arrow indicates the MHV, which is useful as an indicator of the resection margin

the confluence of the veins does not split. In addition, the viewing angle when laparoscopic surgery is performed differs from that of open surgery and has the angular characteristics of approaching the vein from the dorsal side ${ }^{[11]}$. If the working site of the hepatectomy is on the ventral side, the tiny amount of bleeding produced will flow to the dorsal side, keeping the area where the operation is performed dry ${ }^{[10]}$.

Another major advantage of laparoscopic surgery is that laparoscopic magnification allows for the observation of detailed anatomy. Reports on liver resection focusing on Laennec's capsule have recently been published ${ }^{[12]}$. Particularly during dissection of the hepatic vein near the inflow of the vein into the inferior vena cava (IVC), it is useful to be aware of Laennec's capsule and to select the layers of the vein during dissection, as any injury can be critical ${ }^{[13]}$. Kiguchi et al. ${ }^{[14]}$ reported that the layer between the hepatic and cardiac Laennec's capsule around the root of the hepatic veins could be divided and the hepatic veins safely exposed. He advocated an "inter-Laennec approach" that enables liver dissection preserving only the cardiac Laennec's capsule around the vein wall ${ }^{[15]}$. This approach might have the advantage of securing the resection margin when the tumor is close to the hepatic vein. The role of the major hepatic veins is described below.

\section{Middle hepatic vein}

The middle hepatic vein (MHV) is located in the center of the liver, and thus, it is the most important landmark and has a wide range of applications. It runs along the area boundary between the right and left lobes of the liver and is located in a discrete section of the Cantlie line. As a matter of course, it runs along the transection plane of the right or left lobectomy. The MHV serves as a landmark on the right side of the transection plane for left internal sectionectomy and on the left side of the dissected section for right anterior sectionectomy. In addition, during the segmentectomy of S8, when the MHV is exposed from the central side, the Glisson branch of S8 can be identified so as to cross MHV as the method of the 


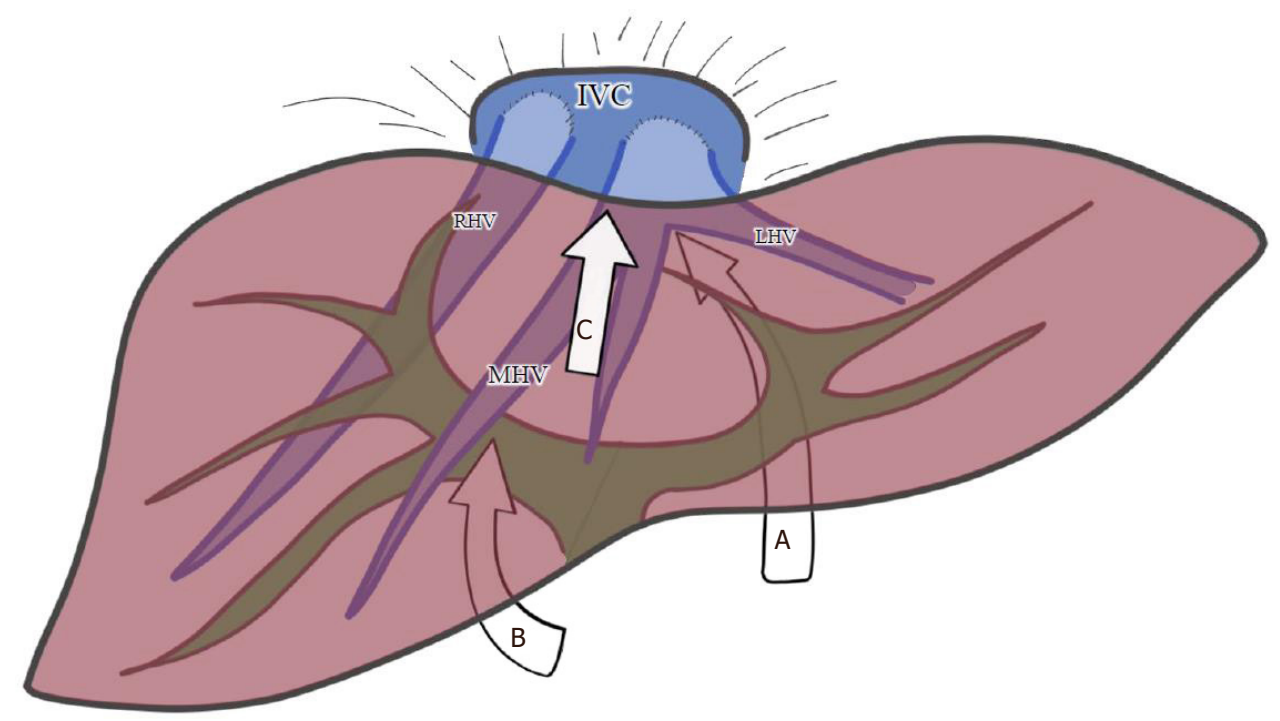

Figure 2. Approaches to MHV. A: dorsal approach; B. hilar approach; C. cranial approach. MHV: middle hepatic vein; RHV: right hepatic vein; LHV: left hepatic vein; IVC: inferior vena cava

intrahepatic "Glissonean approach" ${ }^{[16]}$. The caudal approach is an important conceptual framework for $\operatorname{LLR}^{[10,17,18]}$

Three main approaches to MHV have been reported for MHV [Figure 2]. First, a dorsal approach to the MHV has been described as the approach to the root of $\mathrm{MHV}^{[19]}$. Ome et al. ${ }^{[20]}$ described an "Arantius-first approach" for laparoscopic left hepatecomy. Following the mobilization of the left lateral lobe, the roots of left hepatic vein (LHV) and MHV are exposed in the dorsal view. Upturning the left lateral lobe, the liver parenchyma above Arantius' duct is opened widely between the root of the LHV and left Glissonean pedicle. After dividing the left Glissonean pedicle, the main trunk of the MHV can be exposed in a good dorsal view. The liver parenchyma between the MHV and the demarcation line on the liver surface can be easily resected as this plane is theoretically an intersegmental plane where no major vessels transverse.

Second, as the MHV runs cranial and anterior in close proximity to the portal bifurcation, and owing to the unique laparoscopic caudal view provided, an early identification of the MHV above the hilum at the beginning of the transection ensures a safe and reliable pathway ${ }^{[18]}$. The MHV is exposed immediately after the dissection of the hilar area [Figure 3A and B]. The distance between the MHV and right Glissonean pedicle is 1 to $2 \mathrm{~cm}^{[21]}$. This method is effective for both left and right hemi-hepatectomy. Once the MHV is identified, transection of the liver anterior to the MHV proceeds rapidly along the Cantlie line [Figure 3C].

Third is the cranial approach to the root of the MHV. Although the angle of the surgical device is somewhat awkward, it is relatively easy to expose the ventral side of the MHV root. In liver resections that eventually reach the left or right side of the MHV root (e.g., left hemi-hepatectomy, right hemi-hepatectomy, right anterior sectionectomy and left internal sectionectomy), the transection line can be determined by first setting the goal of liver resection. Monden et al. ${ }^{[22]}$ reported that even in the resection of tumors close to the MHV, an appropriate transection line can be selected by being aware of Laennec's capsule.

\section{Right hepatic vein}

The right hepatic vein (RHV) runs along the boundary between the right posterior section and right anterior section. It becomes a landmark in the right anterior sectionectomy and the landmark on the left side of the right posterior sectionectomy. In addition, it serves as an indicator of the transection boundary 


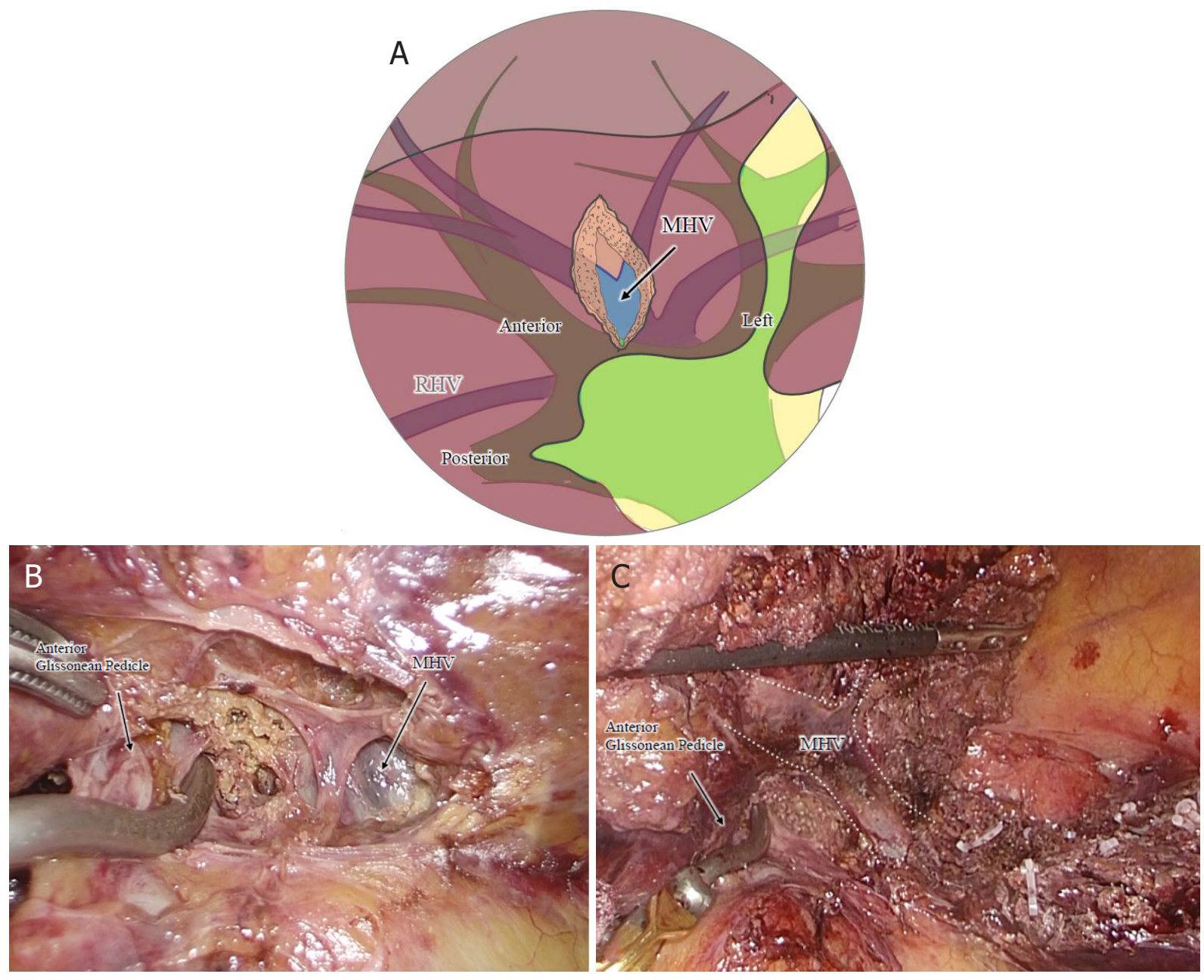

Figure 3. Hilar approach to MHV. A, B: the MHV is exposed immediately after the dissection of the hilar area; C: a case of expanded right anterior sectionectomy that includes the MHV. The exposed MHV provides a good guide to the left resection surface. MHV: middle hepatic vein; RHV: right hepatic vein

when performing a segmental resection such as S7 and S6. However, the exposure of the RHV is deeper than that of the MHV, and the running angle is almost horizontal to dorsal angle, compared to the MHV. Therefore, it requires more ingenuity in its approach.

RHV is a long way from the ventral liver surface in the ventral approach as well as the so-called laparotomy. There are a number of points at which one can easily approach the RHV when taking a caudal approach. Xiao et al. ${ }^{[23]}$ reported that the approach to the RHV root from the head side is easier than from the foot side. However, in some patients, the hepatic parenchyma in front of the RHV root may be raised and the device angle may not be aligned and difficult to approach. Okuda et al. ${ }^{[2]}$ said that even in a poorly accessible right posterior segment, an intercostal trocar makes it easy to perform LLR in a wide operative field. Nevertheless, we believe that the cranial approach depends on the status of the liver according to our experience. We think that it may be difficult to approach in the case of cirrhosis or where the right liver is large because the position of the port and the angle of the device do not match.

On the other hand, the dorsal RHV root approach is a caudal approach that makes use of the characteristics of laparoscopic surgery, and there are some reports [Figure 4]. As an approach for right posterior sectionectomy, Honda et al. ${ }^{[10]}$ proposed a caudate lobe-first approach, a caudal approach technique in 


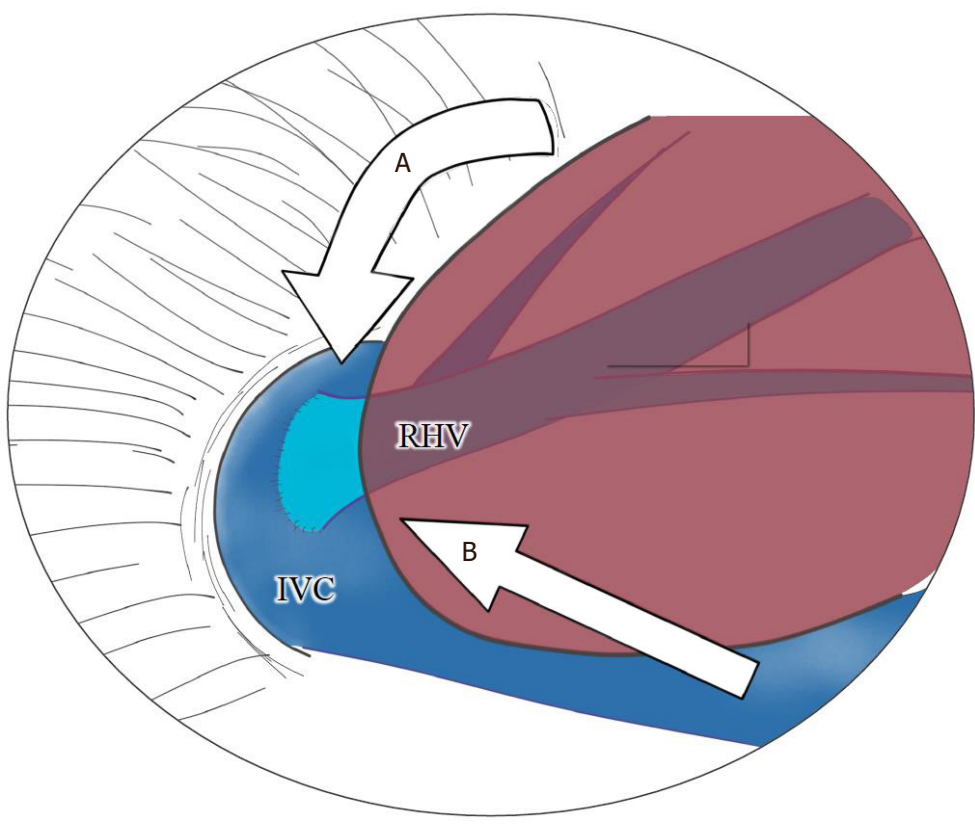

Figure 4. Approaches to RHV. A: cranial approach; B: dorsal approach. RHV: right hepatic vein; IVC: inferior vena cava

which the caudate lobe is transected on the dorsal side of the right Glissonean pedicle. After dividing the posterior Glissonean pedicle, the liver parenchyma of the ventral side of the hepatic hilum is separated by exposing the RHV in the center of the cutting plane. By checking the RHV rising from the IVC at this time, an appropriate hepatic transection plane can be selected. It is relatively easy to expose RHV in the dorsal visual field of the liver, and it has been reported to secure the excision of S7 by making it a characteristic. Ome et al. ${ }^{[25]}$ proposed that when performing segmentectomy of segment 7, the Glissonean pedicle of segment 7 is dissected by the intrahepatic Glissonean approach, and the RHV should then be exposed from the root to the periphery. The parenchyma between the RHV and the demarcation line is transected from the dorsal side. Lee et al. ${ }^{[26]}$ also reported a similar method as hepatic vein-first approach for liver resection in segment 7.

\section{HEPATIC VEIN-GUIDED LIVER RESECTION}

The hepatic vein is also an important indicator of hepatic transection in laparotomy. Laparoscopic surgery is a more dependable anatomical structure because venous bleeding is well controlled. In laparoscopic surgery, the three-dimensional spatial perception is inferior to that of laparotomy. Also, it is a challenge to secure an appropriate transection line, and it is useful to use the hepatic vein as a guidepost for hepatic transection while exposing it. While this review focused on the usefulness of the hepatic vein, another important landmark is naturally the major Glisson's branches. The Glissonean approach also plays a role in liver resection in terms of its effectiveness in performing an anatomical resection and its reproducibility even in LLR, and it can be useful in performing major liver resections that have a long plane of parenchymal transection. These systematic resection planes are composed of anatomical elements such as the hepatic veins, the major Glisson's branches, and the demarcation lines of the liver surface.

There are some objections to this cranial approach, which prioritizes exposure of the hepatic vein. Turco et al. ${ }^{[27]}$ point out two problems. First, the cranial approach can potentially be dangerous because bleeding can occur at the deeper plane of the transection plane, and without control of the common trunk of hepatic veins, bleeding can be difficult to control. The other problem is that, on the principle of oncological resection, the Glissonean pedicles should be divided first, while dissecting the hepatic vein first would 
be the reverse of the order. The hepatic vein has often been used as an indicator of hepatic dissection in anatomical liver resection. However, since anatomical liver resection is performed in the vascular supply area of the portal vein, hepatic vein-guided liver resection may not be synonymous with anatomical resection. In addition, a hepatectomy method using the hepatic vein as a guide has been proposed until now, but the terms have not been unified. In the future, it will be important to demonstrate the usefulness of this technique for hepatectomy using the hepatic vein as a reference point. Also, the terminology for the approach is not entirely consistent. Therefore, it is expected that these procedures will be discussed at consensus conferences in the future.

\section{CONCLUSION}

It should be recognized that the hepatic vein provides a useful structure to guide liver transection for LLR. It is also an anatomical landmark, to a lesser extent, in open hepatectomy. In the planning of hepatic transection, it is recommended that the hepatic vein be utilized as the boundary of the transection or a landmark that serves as an anatomical reference point.

\section{DECLARATIONS}

\section{Authors' contributions}

Manuscript writing and operator of LLR: Ban D

Substantial contributions to conception: Esaki M, Shimada K

Technical supports and interpretation: Nara S, Takemoto T

Performed data acquisition, and drawing the figures: Mizui T, Yoshino J

\section{Availability of data and materials}

Not applicable.

\section{Financial support and sponsorship}

None.

\section{Conflicts of interest}

All authors declared that there are no conflicts of interest.

\section{Ethical approval and consent to participate}

Not applicable.

\section{Consent for publication}

Not applicable.

\section{Copyright}

(c) The Author(s) 2021.

\section{REFERENCES}

1. Ban D, Tanabe M, Kumamaru H, et al. Safe dissemination of laparoscopic liver resection in 27,146 cases between 2011 and 2017 from the national clinical database of Japan. Ann Surg 2020; doi: 10.1097/SLA.0000000000003799.

2. Cho JY, Han HS, Choi Y, et al. Association of remnant liver ischemia with early recurrence and poor survival after liver resection in patients with hepatocellular carcinoma. JAMA Surg 2017;152:386-92.

3. Yamashita S, Venkatesan AM, Mizuno T, et al. Remnant liver ischemia as a prognostic factor for cancer-specific survival after resection of colorectal liver metastases. JAMA Surg 2017;152:e172986.

4. Nomi T, Hokuto D, Yoshikawa T, Matsuo Y, Sho M. A novel navigation for laparoscopic anatomic liver resection using indocyanine green fluorescence. Ann Surg Oncol 2018;25:3982.

5. Urade T, Sawa H, Iwatani Y, et al. Laparoscopic anatomical liver resection using indocyanine green fluorescence imaging. Asian J Surg 
2020;43:362-8.

6. Kubo N, Araki K, Harimoto N, et al. Hepatic resection for the right hepatic vein drainage area with indocyanine green fluorescent imaging navigation. J Hepatobiliary Pancreat Sci 2020;27:371-9.

7. Kobayashi S, Honda G, Kurata M, et al. An experimental study on the relationship among airway pressure, pneumoperitoneum pressure, and central venous pressure in pure laparoscopic hepatectomy. Ann Surg 2016;263:1159-63.

8. Ciria R, Cherqui D, Geller DA, Briceno J, Wakabayashi G. Comparative short-term benefits of laparoscopic liver resection: 9000 cases and climbing. Ann Surg 2016;263:761-77.

9. Fretland AA, Dagenborg VJ, Bjornelv GMW, et al. Laparoscopic versus open resection for colorectal liver metastases: the OSLOCOMET randomized controlled trial. Ann Surg 2018;267:199-207.

10. Honda G, Kurata M, Okuda Y, Kobayashi S, Sakamoto K, Takahashi K. Totally laparoscopic anatomical hepatectomy exposing the major hepatic veins from the root side: a case of the right anterior sectorectomy (with video). J Gastrointest Surg 2014;18:1379-80.

11. Ogiso S, Nomi T, Araki K, et al. Laparoscopy-specific surgical concepts for hepatectomy based on the laparoscopic caudal view: a key to reboot surgeons' minds. Ann Surg Oncol 2015;22 Suppl 3:S327-33.

12. Sugioka A, Kato Y, Tanahashi Y. Systematic extrahepatic Glissonean pedicle isolation for anatomical liver resection based on Laennec's capsule: proposal of a novel comprehensive surgical anatomy of the liver. J Hepatobiliary Pancreat Sci 2017;24:17-23.

13. Monden K, Ohno K, Sadamori H, et al. Histology of the Laennec's capsule around the hepatic veins and how it may guide approaches to laparoscopic anatomic lliver resection. Surg Gastroenterol Oncol 2020;25:74-7.

14. Kiguchi G, Sugioka A, Kato Y, Uyama I. Laparoscopic S7 segmentectomy using the inter-Laennec approach for hepatocellular carcinoma near the right hepatic vein. Surg Oncol 2019;31:132-4.

15. Kiguchi G, Sugioka A, Kato Y, Uyama I. Use of the inter-Laennec approach for laparoscopic anatomical right posterior sectionectomy in semi-prone position. Surg Oncol 2019;29:140-1.

16. Ome Y, Honda G, Doi M, Muto J, Seyama Y. Laparoscopic anatomic liver resection of segment 8 using intrahepatic glissonean approach. $J$ Am Coll Surg 2020;230:e13-20.

17. Soubrane O, Schwarz L, Cauchy F, et al. A conceptual technique for laparoscopic right hepatectomy based on facts and oncologic principles: the caudal approach. Ann Surg 2015;261:1226-31.

18. Rotellar F, Marti-Cruchaga P, Zozaya G, et al. Caudal approach to the middle hepatic vein as a resection pathway in difficult major hepatectomies under laparoscopic approach. J Surg Oncol 2020; doi: 10.1002/jso.26150.

19. Okuda Y, Honda G, Kurata M, Kobayashi S, Sakamoto K. Dorsal approach to the middle hepatic vein in laparoscopic left hemihepatectomy. J Am Coll Surg 2014;219:e1-4.

20. Ome Y, Honda G, Kawamoto Y. Laparoscopic left hemihepatectomy by the arantius-first approach: a video case report. $J$ Gastrointest Surg 2020;24:2180-2.

21. Yu DC, Wu XY, Sun XT, Ding YT. Glissonian approach combined with major hepatic vein first for laparoscopic anatomic hepatectomy. Hepatobiliary Pancreat Dis Int 2018;17:316-22.

22. Monden K, Sadamori H, Hioki M, Takakura N. Consideration of cranial approach to major hepatic veins in laparoscopic anatomic liver resection of segment 8. J Am Coll Surg 2020;231:498-9.

23. Xiao L, Li JW, Zheng SG. Laparoscopic anatomical segmentectomy of liver segments VII and VIII with the hepatic veins exposed from the head side (with videos). J Surg Oncol 2016;114:752-6.

24. Okuda Y, Honda G, Kurata M, Kobayashi S, Sakamoto K, Takahashi K. A safe and valid procedure for pure laparoscopic partial hepatectomy of the most posterosuperior area: the top of segment 7. J Am Coll Surg 2015;220:e17-21.

25. Ome Y, Seyama Y, Doi M. Laparoscopic anatomic resection of segment 7 of the liver using the intrahepatic Glissonean approach from the dorsal side (with video). J Hepatobiliary Pancreat Sci 2020;27:E3-6.

26. Lee B, Cho JY, Choi Y, Yoon YS, Han HS. Laparoscopic liver resection in segment 7: hepatic vein first approach with special reference to sufficient resection margin. Surg Oncol 2019;30:87-9.

27. Turco C, Lim C, Goumard C, Scatton O. Laparoscopic anatomic liver resection of segment 8 using the transfissural glissonean approach: the ton that tung technique revisited. J Am Coll Surg 2020;230:836. 